\title{
SHV Lactamase Engineering Database: a reconciliation tool for SHV b-lactamases in public databases
}

\author{
Quan K Thai, Juergen Pleiss
}

\begin{abstract}
Background: SHV b-lactamases confer resistance to a broad range of antibiotics by accumulating mutations. The number of SHV variants is steadily increasing. $117 \mathrm{SHV}$ variants have been assigned in the SHV mutation table (http://www.lahey.org/Studies/). Besides, information about SHV b-lactamases can be found in the rapidly growing NCBI protein database. The SHV b-Lactamase Engineering Database (SHVED) has been developed to collect the SHV b-lactamase sequences from the NCBI protein database and the SHV mutation table. It serves as a tool for the detection and reconciliation of inconsistencies, and for the identification of new SHV variants and amino acid substitutions.

Description: The SHVED contains 200 protein entries with distinct sequences and 20 crystal structures. 83 protein sequences are included in the both the SHV mutation table and the NCBI protein database, while 35 and 82 protein sequences are only in the SHV mutation table and the NCBI protein database, respectively. Of these 82 sequences, 41 originate from microbial sources, and 22 of them are full-length sequences that harbour a mutation profile which has not been classified yet in the SHV mutation table. 27 protein entries from the NCBI protein database were found to have an inconsistency in SHV name identification. These inconsistencies were reconciled using information from the SHV mutation table and stored in the SHVED.

The SHVED is accessible at http://www.LacED.uni-stuttgart.de/classA/SHVED/. It provides sequences, structures, and a multisequence alignment of SHV b-lactamases with the corrected annotation. Amino acid substitutions at each position are also provided. The SHVED is updated monthly and supplies all data for download.

Conclusions: The SHV b-Lactamase Engineering Database (SHVED) contains information about SHV variants with reconciled annotation. It serves as a tool for detection of inconsistencies in the NCBI protein database, helps to identify new mutations resulting in new SHV variants, and thus supports the investigation of sequence-function relationships of SHV b-lactamases.
\end{abstract}

\section{Background}

Since the application of penicillin to the clinical practice in the 1940s, the effectiveness of b-lactam antibiotics have been reduced drastically [1-3]. One of the main reasons is the hydrolysis of their b-lactam ring by b-lactamases (EC 3.5.2.6) resulting in a loss of function. These enzymes, especially SHV and TEM b-lactamase variants, accumulate mutations gradually $[4,5]$ to resist b-lactam antibiotics and rapidly spread over the world [6-8].

\footnotetext{
* Correspondence: Juergen.Pleiss@itb.uni-stuttgart.de
Institute of Technical Biochemistry, University of Stuttgart, Allmandring 31,

* Correspondence: Juergen.Pleiss@itb.uni-stuttgart.de
Institute of Technical Biochemistry, University of Stuttgart, Allmandring 31, 70569 Stuttgart, Germany
} 70569 stitg

(c) 2010 Thai and Pleiss; licensee BioMed Central Ltd. This is an Open Access article distributed under the terms of the Creative Commons Attribution License (http://creativecommons.org/licenses/by/2.0), which permits unrestricted use, distribution, and reproduction in any medium, provided the original work is properly cited.
SHV b-lactamases belong to class A b-lactamases and have a serine in the active site [9]. The premature protein consists of 286 amino acids. The first 21 amino acids at the $\mathrm{N}$-terminus form the signal sequence and are removed to yield the mature enzyme [10]. SHV b-lactamases were first described in the members of the genus Klebsiella as a narrow-spectrum b-lactamase against penicillin $[6,11]$. Their genes are located either in the bacterial chromosome or on a plasmid [12]. Genes encoding these enzymes have been mutated rapidly and transferred to other Gram-negative bacteria in different geographical regions [6]. Currently, 117 SHV variants have been described. A list of assigned 
SHV variants was compiled and maintained by Jacoby and Bush [13] which is referred further in this paper as "SHV mutation table". Beside the SHV mutation table, sequence information on SHV b-lactamases can also be found in the NCBI protein database [14]. One of the important data sources of the NCBI protein database is the NCBI nucleotide database which is open for submission of new sequences without further validation; therefore it is growing rapidly, but contains inconsistencies. In contrast, the SHV mutation table is manually curated by experts in the b-lactamase field and therefore is widely accepted as a reliable and consistent information source. In the SHV mutation table, each SHV variant is characterized by its name and mutation profile which is a set of amino acid substitutions at certain positions in the sequence. Positions are identified according to the Ambler numbering scheme [15]. To become listed in the SHV mutation table as a new SHV b-lactamase, it must have arisen naturally, is fully sequenced, and harbors a new mutation profile [13]. Therefore, engineered proteins are not considered.

The SHV Engineering Database (SHVED) was built up as a comprehensive inventory by collecting data on SHV b-lactamases from these two databases to facilitate detection of inconsistencies in entries derived from NCBI protein database and to eventually reconcile them, to detect new SHV b-lactamases with novel mutation profiles, and to identify new amino acid positions at which mutations can occur.

\section{Construction and content Construction \\ Development and construction of SHVED}

Amino acid sequence of SHV-1 originated from Klebsiella pneumoniae (GenInfo (GI): 4337048) was used as a seed sequence for building up the SHVED. A BLAST search [16] was performed against the NCBI protein database [14] without filtering of low complexity regions and with a low E-value threshold $\left(10^{-124}\right)$ to prevent the occurrence of TEM lactamases and other non-SHV lactamases in the BLAST results. For each hit in the BLAST result, the GI was extracted and the complete XML entry was downloaded from the NCBI protein database. Information on sequence, position-specific annotations, functional descriptions, and source organism was extracted from the entry and parsed by an automated retrieval system into an in-house developed relational database system [17]. For BLAST results representing protein structures, monomers were extracted from the PDB [18] and deposited as structure entries.

Sequences generated from the annotated mutation profiles deposited in the SHV mutation table [13] were also incorporated into the SHVED. Except for 16 assigned SHVs which were "withdrawn" or "not yet released", 117 assigned SHV sequences were generated and parsed into the SHVED using the available information on amino acid exchanges and the reference sequence SHV-1. On the webpage, the "source organism" of these sequences was set to "Clinical sample" and the data source to "lc' abbreviated from "Lahey Clinic" where the SHV mutation table is hosted.

\section{Identification and naming of SHV $\beta$-lactamase sequences}

Each protein sequence in the SHVED was aligned with SHV-1 using ClustalW [19] to identify its mutation profile. This mutation profile is the set of amino acid exchanges, deletions, and insertions occurring in a certain SHV, e.g. L35Q for the substitution of leucine at position 35 by glutamine. Subsequently, the mutation profile was matched against the mutation profiles listed in the SHV mutation table to identify whether the respective protein sequence is identical to an already assigned SHV. If the mutation profiles were identical, the protein was named accordingly (e.g. "SHV-3"). Otherwise it was named "SHV-like" and its mutation profile was stored. In the case of sequences longer than SHV-1, only the region corresponding to SHV-1 was examined to identify the mutation profile. Amino acid insertions arising inside the protein sequence were annotated, e.g. "-162.1D -162.2R" for the insertion of two residues aspartic acid and arginine after the residue at position 162. The amino acid deletion was annotated with the corresponding residue and position, e.g. "G54-" for the deletion of a glycine at position 54 .

For sequences longer than SHV-1, the number of additional residues was recorded, e.g. " $\mathrm{C}+5$ " for a sequence 5 residues longer at its $C$ terminus. Sequences shorter than SHV-1 were considered as fragments of the respective SHV sequences or the SHV-like sequences, although they were probably named differently in the entry of the source database. The number of missing residues at the $\mathrm{N}$ - and $\mathrm{C}$ - terminus were annotated, e.g. "N-21 C-3" for 21 and 3 residues missing at the $\mathrm{N}$ - and $\mathrm{C}$ - terminus, respectively.

\section{Multisequence alignment and feature annotation}

The annotation information was enriched by performing multisequence alignment using CLUSTALW [19]. Information on secondary structure calculated using DSSP [20] were also included in the SHVED. Individual residues in the sequence as well as in the alignments were numbered according to the standard scheme suggested by Ambler [15]

\section{Reconciliation of data inconsistencies}

A systematic comparison of entries of the NCBI protein database and the SHV mutation table allows a reconciliation of NCBI protein database entries which have an inconsistent annotation. In the SHVED, the wrong name assignment is corrected if its mutation profile is 
already included in SHV mutation table. A sequence with a new mutation profile is stored in the SHVED as new SHV b-lactamase, even if it has been named by the authors by a (wrong) SHV name in the NCBI protein database. A link from the reconciled SHVED entry to the original NCBI protein database entry allows the author of the respective entry to correct an erroneous entry.

\section{Content}

\section{Data content of the SHVED}

452 protein sequence entries from NCBI protein database and 117 protein sequences from SHV mutation table were collected and parsed into the SHVED, resulting in 200 distinct protein entries. 20 crystal structures of 2 SHV b-lactamases (SHV-1 and SHV-2) were stored in the SHVED. 19 crystal structures were from SHV-1 with one or two engineered mutations. Apart from the structure (PDB entry 3D4F) which is full-length sequence, all crystal structures lack the 21 residues of the $\mathrm{N}$-terminal signal sequence. Two protein sequences (PDB entries $2 \mathrm{~A} 3 \mathrm{U}$ and $2 \mathrm{~A} 49$ ) possess 5 and 4 additional residues, respectively, at their $\mathrm{C}$-terminus (Table 1 ).

Table 1 PDB code of crystal structure entries in SHVED and their sequence annotations

\begin{tabular}{|c|c|c|}
\hline Protein & PDB ID & Description \\
\hline \multirow[t]{19}{*}{ SHV-1 } & 3D4FA & structure of SHV-1 \\
\hline & 1ONGA & structure $(\mathrm{N}-21)$ of $\mathrm{SHV}-1$ \\
\hline & 1Q2PA & \\
\hline & 1SHVA & \\
\hline & 1VM1A & \\
\hline & $2 \mathrm{G} 2 \mathrm{UA}$ & \\
\hline & $2 \mathrm{H} 5 \mathrm{SA}$ & \\
\hline & 2ZD8A & \\
\hline & $3 \mathrm{C} 4 \mathrm{OA}$ & \\
\hline & 3С $4 \mathrm{PA}$ & \\
\hline & 1TDGA & $\begin{array}{l}\text { structure (N-21) of SHV-1 with the artificial S130G } \\
\text { mutation and tazobactam [S130G] }\end{array}$ \\
\hline & 1TDLA & \\
\hline & $2 \mathrm{~A} 3 \cup \mathrm{A}$ & $\begin{array}{l}\text { structure }(\mathrm{N}-21 \mathrm{C}+5) \text { of } \mathrm{SHV}-1 \text { with the artificial } \\
\text { E166A mutation and sulbactam [E166A] }\end{array}$ \\
\hline & 2G2WA & $\begin{array}{l}\text { structure (N-21) of SHV-1 with the artificial D104K } \\
\text { mutation [D104K] }\end{array}$ \\
\hline & $2 \mathrm{~A} 49 \mathrm{~A}$ & $\begin{array}{l}\text { structure }(\mathrm{N}-21 \mathrm{C}+4) \text { of SHV-1 with the artificial } \\
\text { E166A mutation and clavulanic acid [E166A] }\end{array}$ \\
\hline & $1 \mathrm{RCJA}$ & $\begin{array}{l}\text { structure (N-21) of SHV-1 with the artificial E166A } \\
\text { mutation [E166A] }\end{array}$ \\
\hline & 2HOTA & $\begin{array}{l}\text { structure (N-21) of SHV-1 with artificial M69V, } \\
\text { E166A mutations [M69V E166A] }\end{array}$ \\
\hline & 2HOYA & \\
\hline & $2 \mathrm{H} 10 \mathrm{~A}$ & \\
\hline SHV-2 & 1 N9BA & structure (N-21) of SHV-2 [G238S] \\
\hline
\end{tabular}

Of the 200 proteins, 35 SHV sequences were derived from SHV mutation table, but not from the NCBI protein database, 82 protein sequences were exclusively found in the NCBI protein database, and 83 protein sequences were accessible in both source databases. In 82 protein sequences found only in the NCBI protein database, there are 41 sequences which originate from microbial sources and harbor a new mutation profile. 22 are full-length sequences (table 2) and 19 are fragments (table 3).

\section{Analysis of amino acid substitutions and substitution positions}

In addition to the amino acid substitutions described in the SHV mutation table [13], 27 new substitution

Table 2 New mutation profiles of full length sequences originating from microorganisms

\begin{tabular}{|c|c|c|}
\hline$\overline{\mathrm{Gl}}$ & Source Organism & Description * \\
\hline 74058441 & Klebsiella pneumoniae & SHV-like $[\mathrm{A} 52 \mathrm{~T}]$ \\
\hline 224223446 & Klebsiella pneumoniae & SHV-like [E166K] \\
\hline 154269503 & Klebsiella pneumoniae & SHV-like $[K 256 R]$ \\
\hline 160948441 & Klebsiella pneumoniae & SHV-like $[\mathrm{N} 253 \mathrm{H}]$ \\
\hline 218091981 & Klebsiella pneumoniae & SHV-like [S2711] \\
\hline 161367444 & Klebsiella pneumoniae & SHV-like [R202S] \\
\hline 218091983 & Klebsiella pneumoniae & SHV-like [D104G] \\
\hline 218091988 & Klebsiella pneumoniae & SHV-like [Y7F G238S] \\
\hline 262044380 & Klebsiella pneumoniae & SHV-like [P252L] \\
\hline 41584432 & Escherichia coli & SHV-like [T235N G238S E240K] \\
\hline 218684515 & Klebsiella pneumoniae & SHV-like [H289L] \\
\hline 51150101 & Klebsiella pneumoniae & SHV-like [R6L G238S E240K] \\
\hline 51105097 & Escherichia coli & \\
\hline 30230495 & Acinetobacter baumannii & $\begin{array}{l}\text { SHV-like [L35Q R191H G238S } \\
\text { E240K] }\end{array}$ \\
\hline 33943602 & Acinetobacter baumannii & $\begin{array}{l}\text { SHV-like [S14Y L35Q G238S } \\
\text { E240K] }\end{array}$ \\
\hline 52630976 & Klebsiella pneumoniae & $\begin{array}{l}\text { SHV-like [L35Q G238S E240K } \\
\text { I260V] }\end{array}$ \\
\hline 41584438 & Escherichia coli & $\begin{array}{l}\text { SHV-like [L35Q T235N G238S } \\
\text { E240K] }\end{array}$ \\
\hline 257359515 & Klebsiella pneumoniae & $\begin{array}{l}\text { SHV-like [T18A A22V L35Q } \\
\text { M129V] }\end{array}$ \\
\hline 41584428 & Escherichia coli & $\begin{array}{l}\text { SHV-like [L35Q T235N G238S } \\
\text { E240K S271N] }\end{array}$ \\
\hline 224979335 & Salmonella enterica & $\begin{array}{l}\text { SHV-like [L35Q K94E G238S } \\
\text { E240K] }\end{array}$ \\
\hline 83596180 & Acinetobacter baumannii & $\begin{array}{l}\text { SHV-like [L35Q G238S E240K } \\
\text { N254D] }\end{array}$ \\
\hline 84380855 & Klebsiella pneumoniae & $\begin{array}{l}\text { SHV-like [Y7F L35Q G238S } \\
\text { E240R] }\end{array}$ \\
\hline 15718691 & Klebsiella pneumoniae & $\begin{array}{l}\text { SHV-like [H96T Y97H -162.1D } \\
-162.2 \mathrm{R}-162.3 \mathrm{~W}-162.4 \mathrm{E} \\
-162.5 T]\end{array}$ \\
\hline
\end{tabular}

*SHV-like: SHV variant with a mutation profile that is not in the SHV mutation table. The mutatation profile is given in square brackets. 
Table 3 Fragments with new mutation profiles

\begin{tabular}{|c|c|c|}
\hline GI & Microbial source & Description * \\
\hline 41584430 & Escherichia coli & fragment (C-1) of SHV-like [L35Q T235N G238S E240K] \\
\hline 40950646 & Escherichia coli & fragment (C-2) of SHV-like [L35Q D233E G238S E240K] \\
\hline 164665324 & Escherichia coli & fragment (C-3) of SHV-like [G238S E240X] \\
\hline 90403947 & $\begin{array}{l}\text { Klebsiella } \\
\text { pneumoniae }\end{array}$ & fragment (C-4) of SHV-like [L35Q G238S E240K E288F] \\
\hline 94502905 & $\begin{array}{l}\text { Klebsiella } \\
\text { pneumoniae }\end{array}$ & fragment (N-1 C-3) of SHV-like [R6E Y7G I8D R9S L35Q H289L] \\
\hline 90403949 & Escherichia coli & fragment (N-2 C-7) of SHV-like [Y7W I8V R9I L10F C11P L35Q G238S E240K] \\
\hline 90403945 & $\begin{array}{l}\text { Klebsiella } \\
\text { pneumoniae }\end{array}$ & fragment (N-9 C-1) of SHV-like [L35Q G238S E240K E288N H289L W290G Q291T] \\
\hline 78333 & Escherichia coli & fragment (N-21) of SHV-like [A140T T141A] \\
\hline 90403951 & $\begin{array}{l}\text { Klebsiella } \\
\text { pneumoniae }\end{array}$ & $\begin{array}{l}\text { fragment (N-10 C+1) of SHV-like [L35Q G238S E240K A284R A285P L286Y I287K E288N H289L W290E Q291P } \\
\text { R292K] }\end{array}$ \\
\hline 157838542 & Escherichia coli & fragment (N-19 C-2) of SHV-like [R43S G238S E240K] \\
\hline 46309198 & $\begin{array}{l}\text { Klebsiella } \\
\text { pneumoniae }\end{array}$ & fragment (N-12 C-11) of SHV-like [P252L] \\
\hline 159138973 & $\begin{array}{l}\text { Klebsiella } \\
\text { pneumoniae }\end{array}$ & fragment (N-23 C-5) of SHV-like [L35Q E168A G251S] \\
\hline 159138975 & $\begin{array}{l}\text { Klebsiella } \\
\text { pneumoniae }\end{array}$ & fragment (N-24 C-5) of SHV-like [L35Q E168A G251S] \\
\hline 56463227 & Escherichia coli & fragment (N-25 C-13) of SHV-like [E89Q G238S E240K] \\
\hline 56463237 & Escherichia coli & fragment (N-25 C-13) of SHV-like [V80M G238S E240K] \\
\hline 56463225 & Escherichia coli & fragment (N-25 C-13) of SHV-like [R43S G238S E240K] \\
\hline 56463229 & Escherichia coli & fragment (N-25 C-13) of SHV-like [R43S E89Q G238S E240K] \\
\hline 56463239 & Escherichia coli & fragment (N-25 C-13) of SHV-like [V44G V80M E89Q G238S E240K G251V M272R] \\
\hline 56463235 & Escherichia coli & fragment (N-25 C-13) of SHV-like [R43S D213A T235P G238S E240K E258D V261L] \\
\hline
\end{tabular}

* SHV-like: SHV variant with a mutation profile that is not in the SHV mutation table.

$\mathrm{N}-\mathrm{x} \mathrm{C}-\mathrm{y}$ : sequence lacks $\times$ amino acids at the $\mathrm{N}$ terminus and $\mathrm{y}$ amino acids at the $\mathrm{C}$ terminus. The mutatation profile is given in square brackets.

positions in protein entries originating from microbial sources have been identified. 11 new substitution positions found in full length sequences (table S1, Additional file 1) and 18 new substitution positions were found in fragments (table S2, Additional file 1), in which 2 new substitution positions could be found both in full length sequences and in fragments (positions 6 and 289). These new substitution positions spread over the complete protein sequence, including the signal peptide and the $\mathrm{C}$-terminus. Most of the substitutions found in full length sequences are located at the protein surface and are distant from the active site, except for T235 and I260 (figure 1). Of the 18 new substitution positions found in fragments, 9 positions are at the $C$ terminus, 4 positions on the protein surface, 3 positions in the protein core, and 2 in the signal peptide (figure 2). Not only the substitution at new positions, but also new amino acid exchanges at already known positions were found. As an example, the protein sequence with GI 259038268 harbors an lysine at the position 252 instead of a proline. In the SHV mutation table, only the substitution P252G is described.

\section{Data inconsistencies}

There are 27 distinct protein entries derived from the NCBI protein database having inconsistent annotations (table 4). In all cases, the annotated SHV name is inconsistent with its mutation profile. For example, the protein sequence with GI 40950644 has three mutations (L35Q, G238S, and E240K), therefore, it should be named "SHV-12" according to the SHV mutation table, but it is actually annotated as "betalactamase SHV-5" in the NCBI protein database. In 12 cases, the protein sequence is a fragment and therefore there is not enough information to rename it in the SHVED.

\section{Utility}

A multisequence alignment of all 200 protein entries was generated using CLUSTALW. For protein structures, all sequence entries were included and displayed with aligned secondary structure information. Proteins were labeled by the GIs and linked to the NCBI protein database. Annotation of individual residues is visualized by color-coding in the alignment and upon moving the 


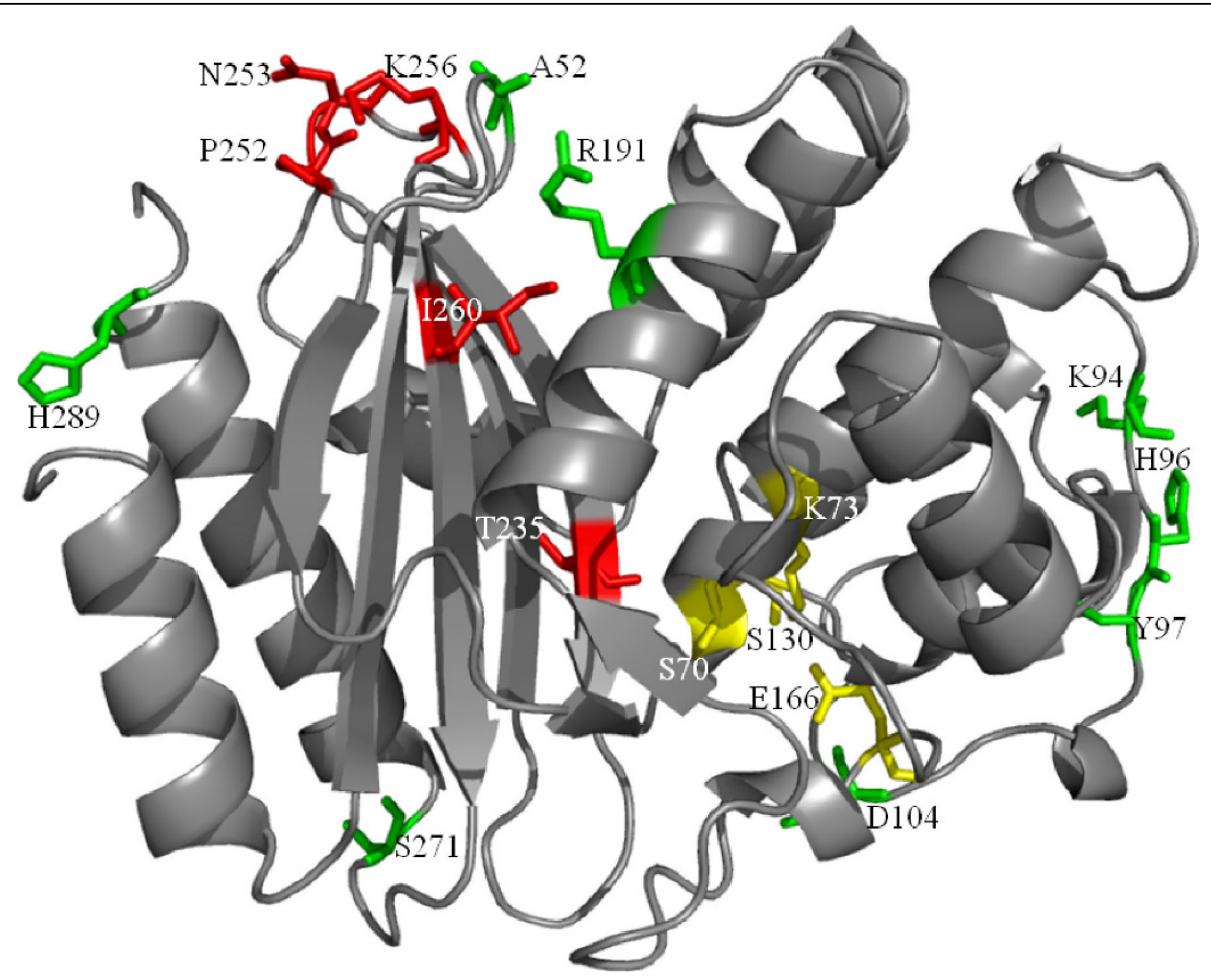

Figure 1 The structure of SHV-1 $\beta$-lactamases (PDB entry 1SHV) with new substitution positions found in full length sequences. Amino acid side chains are shown in stick representation: substitutions occurring at novel positions (green), novel amino acid substitution at known position (red), active site residues (yellow).

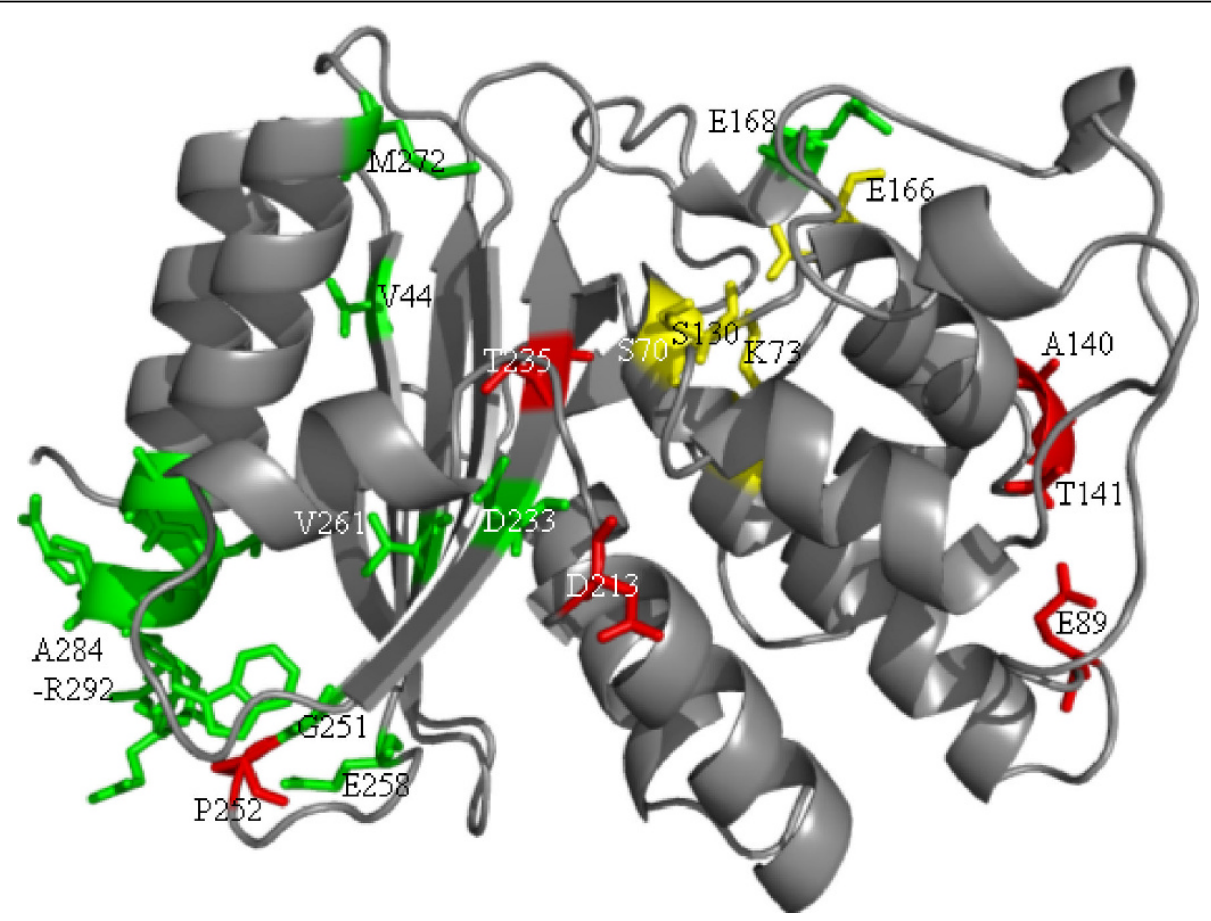

Figure 2 The structure of SHV-1 $\beta$-lactamases (PDB entry 1SHV) with new substitution positions found in fragments. Amino acid side chains are shown in stick representation: substitutions occurring at novel positions (green), novel amino acid substitution at known position (red), active site residues (yellow). 
Table 4 Inconsistencies between information from NCBI protein database and SHV mutation table

\begin{tabular}{|c|c|c|c|}
\hline \multirow[t]{2}{*}{ GI } & \multicolumn{2}{|r|}{ Name and mutation profile according to } & \multirow[t]{2}{*}{ Inconsistency } \\
\hline & NCBI protein database & SHV mutation table* & \\
\hline 262044380 & SHV-5 [G238S E240K] & SHV-like [P252L] & different mutation profile \\
\hline 41584432 & SHV-5 [G238S E240K] & SHV-like [T235N G238S E240K] & \\
\hline 51105097 & SHV-5 [G238S E240K] & SHV-like [R6L G238S E240K] & \\
\hline 40950644 & SHV-5 [G238S E240K] & SHV-12 [L35Q G238S E240K] & \\
\hline 41584438 & SHV-5 [G238S E240K] & SHV-like [L35Q T235N G238S E240K] & \\
\hline 41584428 & SHV-5 [G238S E240K] & SHV-like [L35Q T235N G238S E240K S271N] & \\
\hline 41584434 & SHV-5 [G238S E240K] & fragment (C-1) of SHV-12 [L35Q G238S E240K] & $\begin{array}{l}\text { a fragment with different } \\
\text { mutation profile }\end{array}$ \\
\hline 41584430 & SHV-5 [G238S E240K] & fragment (C-1) of SHV-like [L35Q T235N G238S E240K] & \\
\hline 40950646 & SHV-5 [G238S E240K] & fragment (C-2) of SHV-like [L35Q D233E G238S E240K] & \\
\hline 157838542 & SHV-7 [18F R43S G238S E240K] & fragment (N-19 C-2) of SHV-like [R43S G238S E240K] & \\
\hline 77702548 & SHV-11 [L35Q] & SHV-1 (no mutation) & different mutation profile \\
\hline 90403947 & SHV-12 [L35Q G238S E240K] & fragment (C-4) of SHV-like [L35Q G238S E240K E288F] & $\begin{array}{l}\text { a fragment with different } \\
\text { mutation profile }\end{array}$ \\
\hline 90403949 & SHV-12 [L35Q G238S E240K] & $\begin{array}{l}\text { fragment (N-2 C-7) of SHV-like [Y7W I8V R9I L10F C11P L35Q G238S } \\
\text { E240K] }\end{array}$ & \\
\hline 90403945 & SHV-12 [L35Q G238S E240K] & $\begin{array}{l}\text { fragment (N-9 C-1) of SHV-like [L35Q G238S E240K E288N H289L W290G } \\
\text { Q291T] }\end{array}$ & \\
\hline 90403951 & SHV-12 [L35Q G238S E240K] & $\begin{array}{l}\text { fragment (N-10 C+1) of SHV-like [L35Q G238S E240K A284R A285P L286Y } \\
\text { I287K E288N H289L W290E Q291P R292K] }\end{array}$ & \\
\hline 15718691 & $\begin{array}{l}\text { SHV-16 [-162.1D }-162.2 R \\
-162.3 W-162.4 \mathrm{E}-162.5 T]\end{array}$ & SHV like [H96T Y97H -162.1D -162.2R -162.3W -162.4E -162.5T] & different mutation profile \\
\hline 255045865 & $\mathrm{SHV}-28[\mathrm{Y} 7 \mathrm{~F}]$ & fragment (N-6 C-20) of SHV-1 & $\begin{array}{l}\text { a fragment with different } \\
\text { mutation profile }\end{array}$ \\
\hline 30230495 & SHV-48 [V119l] & SHV-like [L35Q R191H G238S E240K] & different mutation profile \\
\hline 33943602 & SHV-56 [L35Q K234R] & SHV-like [S14Y L35Q G238S E240K] & \\
\hline 83596180 & SHV-71 [H112Y A146V] & SHV-like [L35Q G238S E240K N254D] & \\
\hline 84380855 & SHV-86 [L35Q G238S E240R] & SHV-like [Y7F L35Q G238S E240R] & \\
\hline 159138975 & SHV-102 [G238A] & fragment (N-24 C-5) of SHV-like [L35Q E168A G251S] & $\begin{array}{l}\text { a fragment with different } \\
\text { mutation profile }\end{array}$ \\
\hline 159138973 & SHV-102 [G238A] & fragment (N-23 C-5) of SHV-like [L35Q E168A G251S] & \\
\hline 154269503 & SHV-103 [L250R] & SHV-like $[K 256 R]$ & different mutation profile \\
\hline 161367444 & SHV-104 [M5L R202S] & SHV-like [R202S] & \\
\hline 257359515 & $\begin{array}{l}\text { SHV-121 [18A A22V L35Q } \\
\text { M129V] }\end{array}$ & SHV-like [T18A A22V L35Q M129V] & \\
\hline 256862196 & $\begin{array}{l}\text { SHV-123 [L35Q G238S E240K } \\
\text { P252G N254I] }\end{array}$ & fragment (C-15) of SHV-like [L35Q G238S E240K P252G N255I] & $\begin{array}{l}\text { a fragment with different } \\
\text { mutation profile }\end{array}$ \\
\hline
\end{tabular}

* SHV-like: SHV variant with a mutation profile that is not in the SHV mutation table.

cursor over the respective residue. The SHVED is accessible at http://www.LacED.uni-stuttgart.de/classA/ SHVED by a JavaScript-enabled WWW browser. Protein tables provide information on the protein name, mutation, number of residues missing at the $\mathrm{N}$ - and $\mathrm{C}$-terminal (in case of fragments), and on the source organism. As an alternative to the multisequence alignment, the SHV variants are visualized as mutations relative to the sequence of SHV-1. Substitution positions are colored and annotated by the exchanged amino acids.

\section{Discussion}

\section{Data content of the SHVED}

By systematic analysis of protein sequences in the SHVED, 41 protein sequences with a new mutation profile were identified. 22 of them are full length sequences originating from microbial sources and therefore are candidates for a new SHV number assignment. The new mutations occurred either at new position on the sequence or they were new amino acid exchange at already described positions. 


\section{Detection of novel SHV $\beta$-lactamases and novel amino acid substitutions}

Except for one new mutation profile originating from a synthetic construct (GI 151861), all new mutation profiles originated from microbial sources. As a plasmidbound gene, the SHV b-lactamase encoding bla $\mathrm{SHV}_{\mathrm{SH}}$ genes are easily transferred among the members of Gram-negative bacteria, especially Enterobacteriaceae because of their close genetic relationship [6]. Thus, most of the newly detected SHV b-lactamases are from Enterobacteriaceae such as Klebsiella pneumoniae (14 SHVs), Escherichia coli (15 SHVs), Enterobacter cloacae (1 SHV), from both K.pneumoniae and E.coli (1 SHV), and from both K.pneumoniae and E.cloacae (1 SHV). Additionally, 3 new SHV variants were found in Acinetobacter baumannii and 1 new SHV variant was found in Salmonella enterica. Although 19 fragments harbor a new mutation profile, they can not be assigned to a new SHV number because of missing sequence information. However, the information about the substitution at new positions found in these fragments could be used in the future to predict the occurrence of new SHV variants.

\section{Data inconsistencies and reconciliation}

In all 27 cases of inconsistency, the annotated name differed from the actual mutation profile. However, the reasons of the inconsistency varied. In the case of the protein sequence with GI 154269503, the lysine at position 256 is substituted by an arginine, while it is reported that the lysine is exchanged by an arginine at position 250 (K250R) [21]. In the SHV mutation table, it is listed as SHV-103 and characterized by the substitution of a leucine at position 250 by an arginine (L250R). A mutation at position 256 is not yet recorded in the SHV mutation table, and the mutation at position 250 can only be seen in the SHV-103. Probably, the difference in amino acid numbering by the author of GI 154269503 and by the curators of the SHV mutation table at Lahey Clinic caused the inconsistence. In the case of the protein sequence with GI 161367444, the inconsistency might derive from the primer used. In the sequence, only one mutation R202S was found, while it is annotated as SHV-104 which has two mutations (M5L and R202S) according to the SHV mutation table. It is noted in the NCBI entry that the forward primer "ATGCGTTATATTCGCCTGTGTATT" was used to amplify the target DNA, which results a methionine at position 5 . Therefore, the deduced amino acid substitution M5L (if it actually occurred) could not be present in the deposited amino acid sequence, and the deposited amino acid sequence should not be annotated as SHV-104 because it does not harbor the mutation profile 'M5L R202S'. In the case of the protein sequence with GI 15718691, the duplication of a pentapeptide 163DRWET167 was reported [22] and assigned as SHV-16. But in addition, two mutations $\mathrm{H} 96 \mathrm{~T}$ and $\mathrm{Y} 97 \mathrm{H}$ are present in the amino acid sequence. Therefore, it is not clear whether the actual SHV-16 harbors only the pentapeptide duplication or additionally the mutations $\mathrm{H} 96 \mathrm{~T}$ and $\mathrm{Y} 97 \mathrm{H}$. In other cases of inconsistency, the amino acid sequences were submitted to the NCBI protein database without corresponding publication and showed inconsistencies in their annotation. One example is the protein sequence with GI 30230495. It is annotated as SHV-48 which should harbor mutation V119I according to the SHV mutation table, while actually four mutations (L35Q, R191H, G238S, and E240K) were found in the deposited amino acid sequence. In the SHV mutation table, an inconsistency in residue numbering (position 253 and 255) was revealed and communicated to the curator for correction.

\section{Conclusion}

The SHV Lactamase Engineering Database (SHVED) was established to identify new SHV b-lactamases and to identify inconsistencies in public databases. Based on our analysis, 22 candidates for assignment of new SHV names were identified. 27 proteins entries with inconsistencies were found and reconciled. Also, three assigned mutation profiles were identified to be in doubt: SHV-16, SHV-103, and SHV-104. The SHVED thus supports the scientific community to name new SHV b-lactamases and to reconcile existing annotation of SHV b-lactamases sequences.

\section{Availability and requirements}

The SHVED is accessible at http://www.LacED.uni-stuttgart.de/classA/SHVED/ by a JavaScript-enabled WWW browser.

\section{Additional material}

Additional file 1: Additional_file_1.pdf contains table S1 and table

S2 mentioned in the text. They list new mutation profiles of sequences derived from microbial organisms.

\section{Abbreviations}

Gl: Genlnfo Identifier; SHVED: SHV Engineering Database.

\section{Acknowledgements}

We acknowledge Florian Wagner for valuable discussions and his support in building up the database. This work was supported by the Federal Ministry of Education and Research of Germany (VNB 04/B12).

\section{Authors' contributions}

QKT developed the database, built the web pages, analyzed the data, and drafted the manuscript. JP supervised the study and finalized the manuscript. All authors read and approved the final manuscript.

Received: 27 April 2010 Accepted: 13 October 2010 Published: 13 October 2010 
1. Livermore DM: beta-Lactamases in laboratory and clinical resistance. Clin Microbiol Rev 1995, 8(4):557-584.

2. Paterson DL, Bonomo RA: Extended-spectrum beta-lactamases: a clinical update. Clin Microbiol Rev 2005, 18(4):657-686.

3. Bradford PA: Extended-spectrum beta-lactamases in the 21 st century: characterization, epidemiology, and detection of this important resistance threat. Clin Microbiol Rev 2001, 14(4):933-951, table of contents.

4. Gniadkowski M: Evolution of extended-spectrum beta-lactamases by mutation. Clin Microbiol Infect 2008, 14(1):11-32.

5. Hall BG, Barlow M: Evolution of the serine beta-lactamases: past, present and future. Drug Resist Updat 2004, 7(2):111-123.

6. Heritage J, M'Zali FH, Gascoyne-Binzi D, Hawkey PM: Evolution and spread of SHV extended-spectrum beta-lactamases in Gram-negative bacteria. Journal of Antimicrobial Chemotherapy 1999, 44(3):309-318.

7. Paterson DL, Hujer KM, Hujer AM, Yeiser B, Bonomo MD, Rice LB, Bonomo RA: Extended-spectrum beta-lactamases in Klebsiella pneumoniae bloodstream isolates from seven countries: dominance and widespread prevalence of SHV- and CTX-M-type beta-lactamases. Antimicrob Agents Chemother 2003, 47(11):3554-3560.

8. Chang FY, Siu LK, Fung CP, Huang MH, Ho M: Diversity of SHV and TEM beta-lactamases in Klebsiella pneumoniae: gene evolution in Northern Taiwan and two novel beta-lactamases, SHV-25 and SHV-26. Antimicrob Agents Chemother 2001, 45(9):2407-2413.

9. Ambler RP: The structure of beta-lactamases. Philos Trans $R$ Soc Lond $B$ Biol Sci 1980, 289(1036):321-331.

10. Kuzin AP, Nukaga M, Nukaga $Y$, Hujer AM, Bonomo RA, Knox JR: Structure of the SHV-1 beta-lactamase. Biochemistry 1999, 38(18):5720-5727.

11. Nugent ME, Hedges RW: The nature of the genetic determinant for the SHV-1 beta-lactamase. Mol Gen Genet 1979, 175(3):239-243.

12. Ford PJ, Avison MB: Evolutionary mapping of the SHV beta-lactamase and evidence for two separate IS26-dependent blaSHV mobilization events from the Klebsiella pneumoniae chromosome. J Antimicrob Chemother 2004, 54(1):69-75.

13. Jacoby G, Bush K: SHV Extended-Spectrum and Inhibitor Resistant BLactamases. [http://www.lahey.org/Studies/]

14. Benson DA, Karsch-Mizrachi I, Lipman DJ, Ostell J, Wheeler DL: GenBank. Nucleic Acids Res 2008, 36(Database):D25-30

15. Ambler RP, Coulson AF, Frere JM, Ghuysen JM, Joris B, Forsman M, Levesque RC, Tiraby G, Waley SG: A standard numbering scheme for the class A beta-lactamases. Biochem J 1991, 276(Pt 1):269-270.

16. Altschul SF, Madden TL, Schaffer AA, Zhang J, Zhang Z, Miller W, Lipman DJ: Gapped BLAST and PSI-BLAST: a new generation of protein database search programs. Nucleic Acids Res 1997, 25(17):3389-3402.

17. Fischer M, Thai QK, Grieb M, Pleiss J: DWARF-a data warehouse system for analyzing protein families. BMC Bioinformatics 2006, 7:495.

18. Schwede T, Diemand A, Guex N, Peitsch MC: Protein structure computing in the genomic era. Res Microbiol 2000, 151(2):107-112.

19. Thompson JD, Higgins DG, Gibson TJ: CLUSTAL W: improving the sensitivity of progressive multiple sequence alignment through sequence weighting, position-specific gap penalties and weight matrix choice. Nucleic Acids Res 1994, 22(22):4673-4680.

20. Kabsch W, Sander C: Dictionary of Protein Secondary Structure - PatternRecognition of Hydrogen-Bonded and Geometrical Features. Biopolymers 1983, 22(12):2577-2637.

21. Abbassi MS, Torres C, Achour W, Vinue L, Saenz Y, Costa D, Bouchami O, Ben Hassen A: Genetic characterisation of CTX-M-15-producing Klebsiella pneumoniae and Escherichia coli strains isolated from stem cell transplant patients in Tunisia. International Journal of Antimicrobial Agents 2008, 32(4):308-314.

22. Arpin C, Labia R, Andre C, Frigo CC, El Harrif Z, Quentin C: SHV-16, a betalactamase with a pentapeptide duplication in the omega loop. Antimicrobial Agents and Chemotherapy 2001, 45(9):2480-2485.

\section{doi:10.1186/1471-2164-11-563}

Cite this article as: Thai and Pleiss: SHV Lactamase Engineering

Database: a reconciliation tool for SHV b-lactamases in public databases. BMC Genomics 2010 11:563.

\section{Submit your next manuscript to BioMed Central and take full advantage of:}

- Convenient online submission

- Thorough peer review

- No space constraints or color figure charges

- Immediate publication on acceptance

- Inclusion in PubMed, CAS, Scopus and Google Scholar

- Research which is freely available for redistribution

Submit your manuscript at www.biomedcentral.com/submit 